\title{
The natural parameterization of cosmic neutrino oscillations
}

\author{
Andrea Palladino $^{1, \mathrm{a}}$, Francesco Vissani $^{1,2, \mathrm{~b}}$ \\ ${ }^{1}$ Gran Sasso Science Institute, INFN, L'Aquila, AQ, Italy \\ ${ }^{2}$ Laboratori Nazionali del Gran Sasso, INFN, Assergi, AQ, Italy
}

Received: 5 August 2015 / Accepted: 6 September 2015 / Published online: 16 September 2015

(c) The Author(s) 2015. This article is published with open access at Springerlink.com

\begin{abstract}
The natural parameterization of vacuum oscillations in three neutrino flavors is studied. Compact and exact relations of its three parameters with the ordinary three mixing angles and $\mathrm{CP}$-violating phase are obtained. Its usefulness is illustrated by considering various applications: the study of the flavor ratio and of its uncertainties, the comparison of expectations and observations in the flavor triangle, and the intensity of the signal due to Glashow resonance. The results in the literature are easily reproduced and in particular the recently obtained agreement of the observations of IceCube with the hypothesis of cosmic neutrino oscillations is confirmed. It is argued that a Gaussian treatment of the errors appropriately describes the effects of the uncertainties on the neutrino oscillation parameters.
\end{abstract}

\section{Contents}

1 Introduction . . . . . . . . . . . . . . 1

2 Natural parameters for three flavor vacuum oscillations . 2

2.1 Motivations and definition . . . . . . . . 2

2.2 Connection with the standard parameters of neutrino mixing ........... 3

2.3 Numerical analysis . . . . . . . . . . 3

2.4 Comparison with other parameterizations . . . 3

3 Applications ................. 4

3.1 Flavor ratio after the oscillations $\ldots \ldots \ldots 5$

3.2 Errors on flavor ratio . . . . . . . . . . 6

3.3 Glashow resonance . . . . . . . . . . . 6

4 Summary and discussion . . . . . . . . . . 7

Appendix A: Allowed regions in the Gaussian

approximation ............. 8

References ............... . . 8

a e-mail: andrea.palladino@gssi.infn.it

be-mail: vissani@lngs.infn.it

\section{Introduction}

After the IceCube results, see e.g. [1-3], the importance of a precise description of oscillations has increased greatly. In the present paper we discuss a natural, easy-to-use, and completely general choice of the relevant parameters. We illustrate its usefulness by quantifying the impact of the uncertainties on various physical quantities, implied by the imprecise knowledge on oscillations.

We begin by recalling the main achievements in the discussion of cosmic neutrino oscillations. The general formula for the vacuum averaged oscillations was given in [4]. Ref. [5] studied for the first time the implications of the observed oscillation phenomena on cosmic neutrinos. Various authors remarked the possibility to measure flavor ratios, possibly aiming to constrain the parameters of oscillations, e.g. [69]. The relevance of oscillations for the interpretation of Glashow resonance was noted in [10,11]. In [12] the single parameter that determines cosmic neutrino oscillations and depends linearly upon unknown quantities was identified; then it was remarked [13] that this leads to a strong correlation between the effect of the oscillation parameters on the probabilities of oscillation, lessening chances of measuring the oscillation parameters. Non-linear effects were studied in various subsequent papers including [14-16]. An interesting expression for all relevant parameters of vacuum oscillations was proposed in [17] within a scheme of approximation aimed to improve the tribimaximal mixing matrix. In [17-19] an expansion in second order of small parameters of this parameterization was obtained and applied to the study of cosmic neutrinos. The consistency of vacuum oscillations and IceCube observations was discussed in [20-22]; compare with [23]. In [21] the impact on the flavor ratio of the uncertainties on oscillation parameters was analyzed and the present work develops the discussion.

In this work, we show that the parameterization of neutrino oscillations in vacuum introduced in [17] can be promoted to an exact parameterization and can be argued to be the 
natural parameterization for the discussion of oscillations of cosmic neutrinos. ${ }^{1}$ We obtain new and exact expressions of the three parameters in terms of the known mixing angles and CP-violating phase. We evaluate their numerical values and uncertainties, illustrating their usefulness by discussing three applications: (1) we compare the predicted flavor fractions and those that are allowed by the present observations; (2) we quantify the uncertainties in the prediction of the fraction of muon neutrinos due to oscillations; (3) we argue that, even after accounting very conservatively for the uncertainties of oscillations, the intensity of the Glashow resonance [25] differs greatly in the alternative cases of $p p$ - and $p \gamma$ production as remarked in [10] and later discussed in [18,2628]. Throughout this work, we argue that a Gaussian treatment of the errors of these natural parameters is quite adequate for the present precision.

\section{Natural parameters for three flavor vacuum oscillations}

In this section, we motivate, define and analyze a parameterization of vacuum neutrino oscillations, elucidating the relationship between this and other parameterizations.

We begin by counting the number of independent vacuum oscillation parameters [4]

$P_{\ell \ell^{\prime}}=\sum_{i=1}^{n}\left|U_{\ell i}^{2}\right|\left|U_{\ell^{\prime} i}^{2}\right| \quad$ where $\ell=e, \mu, \tau, \ldots$

in the case of $n$ light neutrinos.

The vacuum oscillation formula depends upon the squares of the leptonic mixing matrix $\left|U_{\ell i}^{2}\right|$. These correspond to $(n-1)^{2}$ independent parameters, as is clear considering all $\left|U_{\ell i}^{2}\right|$ as independent (albeit constrained) parameters except the ones of the first row and column, which can be obtained from unitarity, e.g., $\left|U_{\mu 1}^{2}\right|=1-\sum_{i=2}^{n}\left|U_{\mu i}^{2}\right|$.

But the vacuum oscillation probabilities $P_{\ell \ell^{\prime}}$ are also symmetric in the exchange of the flavor indices $\ell \leftrightarrow \ell^{\prime}$, thus they require less parameters. Since we can again rely on similar unitarity relations when we sum on all flavors, namely $\sum_{\ell} P_{\ell \ell^{\prime}}=1$, this implies that the number of independent parameter is just $n(n-1) / 2$. This means that when $n=3$ we have three independent parameters, when $n=4$ we have six of them, etc.

From now on and in view of the present experimental situation, we focus on the three flavor case $(n=3)$ where we have three parameters, as first remarked (to the best of our knowledge) in Ref. [17].

\footnotetext{
${ }^{1}$ An alternative choice of the parameterization that gives different insight on the allowed ranges of the oscillation probabilities is discussed in [24].
}

\subsection{Motivations and definition}

Next, we motivate and introduce the choice of the three natural parameters. The parameters $P_{0}, P_{1}, P_{2}$ are defined as follows:

$$
\begin{gathered}
P_{0}=\frac{P_{e e}-\frac{1}{3}}{2}, \quad P_{1}=\frac{P_{e \mu}-P_{e \tau}}{2}, \\
P_{2}=\frac{P_{\mu \mu}+P_{\tau \tau}-2 P_{\mu \tau}}{4} .
\end{gathered}
$$

We can write in terms of $P_{0}, P_{1}, P_{2}$ the matrix that contains the probabilities of oscillations of cosmic neutrinos. This is the following symmetric matrix:

$\mathcal{P}=\left(\begin{array}{ccc}\frac{1}{3}+2 P_{0} & \frac{1}{3}-P_{0}+P_{1} & \frac{1}{3}-P_{0}-P_{1} \\ & \frac{1}{3}+\frac{P_{0}}{2}-P_{1}+P_{2} & \frac{1}{3}+\frac{P_{0}}{2}-P_{2} \\ & \frac{1}{3}+\frac{P_{0}}{2}+P_{1}+P_{2}\end{array}\right)$.

It acts on the vector of fluxes before oscillations $F^{0}=$ $\left(F_{e}^{0}, F_{\mu}^{0}, F_{\tau}^{0}\right)$ just as $F=\mathcal{P} F^{0}$, giving the vector of fluxes observed after oscillations, $F=\left(F_{e}, F_{\mu}, F_{\tau}\right)$.

We would like to give our reasons (which are largely based on the available experimental information) why we consider Eq. 2 to be the optimal choice of parameters.

1. The oscillation probability that is singled out in $P_{0}$ is $P_{e e}$, which is well known (being directly measured by low-energy solar neutrino experiments and probed also by reactor and high-energy solar neutrino experiments).

2. The difference of $P_{e \mu}-P_{e \tau}$ contains most of the uncertainties.

3. The last combination of oscillations probabilities, $P_{2}$, is positive and very small.

4. A specific choice of the overall coefficients is adopted in order to have coefficients that are either zero or close to 1 in the expressions of all oscillation probabilities, Eq. 3.

5. Setting $P_{0}=P_{1}=P_{2}=0$, all oscillation probabilities become $P_{\ell \ell^{\prime}}=1 / 3$, namely, any information on the original flavor is lost: the three parameters describe the potentially measurable information on flavor that survives cosmic neutrino oscillations.

The above argument clarifies that it is possible to introduce such a parameterization directly, without the need to associate it to a specific scheme of approximation of the mixing matrix, but rather, keeping it exact. However, various approximation schemes used in the literature have allowed us to uncover the most interesting properties: The second one is known since [12] while the first evidence of the third one was found in [17]. A detailed comparison with other parameterizations used in the literature is presented in Sect. 2.4. 
2.2 Connection with the standard parameters of neutrino mixing

Compact and useful expressions of the natural parameters in terms of four standard parameters, the mixing angles $\theta_{12}, \theta_{23}, \theta_{13}$, and the CP phase violation phase $\delta$, are as follows:

$$
\begin{aligned}
& P_{0}=\frac{1}{2}\left\{\left(1-\epsilon^{2}\right)\left[1-\frac{\sin ^{2} \theta_{12}}{2}\right]+\epsilon^{2}-\frac{1}{3}\right\} \\
& P_{1}=\frac{1-\epsilon}{2}\left\{\gamma \cos 2 \theta_{12}+\beta \frac{1-3 \epsilon}{2}\right\} \\
& P_{2}=\frac{1}{2}\left\{\gamma^{2}+\frac{3}{4} \beta^{2}(1-\epsilon)^{2}\right\}
\end{aligned}
$$

where we introduce for convenience the following four small parameters:

$\epsilon=\sin ^{2} \theta_{13}, \quad \alpha=\sin \theta_{13} \cos \delta \sin 2 \theta_{12} \sin 2 \theta_{23}$,

$\beta=\cos 2 \theta_{23}, \quad \gamma=\alpha-\frac{\beta}{2} \cos 2 \theta_{12}(1+\epsilon)$.

These expressions are new and exact. Note property 3 listed in Sect. 2.1 of this parameterization.

\subsection{Numerical analysis}

The parameters $\alpha, \beta, \gamma$ are small and to date not known precisely, whereas $\epsilon$ is very small and precisely known. We can then order these parameters according to their (presumed) size, and consider $\sin ^{2} 2 \theta_{12}$ to be of zeroth order; $\sin \theta_{13}$, $\cos 2 \theta_{23}, \alpha, \beta, \gamma$ of first order; $\epsilon$ of second order. In the same sense, $P_{0}$ is a zeroth-order parameter; $P_{1}$ is of first order in $\alpha$ and $\beta ; P_{2}$ is second order in $\alpha$ and $\beta$. Note that $P_{2}$ is bound to be positive.

Using the present knowledge of the mixing angle and CPviolating phase [29], we obtain the values and the errors of the natural parameters. We show the results in the Table 1, assuming normal mass hierarchy. It is easy to repeat the same steps with inverted hierarchy, but the differences are not large. From this table we notice that with present data the average values obey $\left\langle P_{0}\right\rangle \gg\left\langle P_{1}\right\rangle \simeq\left\langle P_{2}\right\rangle$ whereas their variances obey $\delta P_{1} \gg \delta P_{0} \simeq \delta P_{2} . P_{0}$ is well known, because it is related to survival probability of solar low-energy neutrinos and $\theta_{13}$ or $\epsilon$ is well measured by reactor experiments. As we see from Fig. $1, P_{0}$ and $P_{1}$ are well represented by Gaussian functions; $P_{2}$ is not Gaussian but it is a very small parame-

Table 1 Table of present values and errors of the natural parameters

\begin{tabular}{lll}
\hline Parameter & Mean value & Standard deviation \\
\hline$P_{0}$ & 0.109 & 0.005 \\
$P_{1}$ & 0.000 & 0.029 \\
$P_{2}$ & 0.010 & 0.007 \\
\hline
\end{tabular}

ter. For these reasons, as we argue in the rest of this work, we can use a Gaussian approximation without introducing severe inaccuracies in the numerical analysis of the oscillations. This is a new result, which allows one to obtain convenient analytical expressions for different examined quantities and to easily quantify the uncertainties.

The probabilities of oscillation given in Eq. 3 have a very simple form: they depend linearly upon the natural parameters. Moreover, in first approximation, they could be expressed only in terms of $P_{0}$, because $P_{1}$ and $P_{2}$ give small corrections. Using the value of Table 1 and the natural parameterization of oscillation matrix, we obtain the probabilities of the oscillations,

$P_{e e}=0.552 \pm 0.010, \quad P_{e \mu}=P_{e \tau}=0.224 \pm 0.029$,

Two couples of probabilities have (almost) the same values, because with the present best fit value $\left\langle P_{1}\right\rangle=0$ and the numerical differences between these expressions are small.

\subsection{Comparison with other parameterizations}

First of all, we consider the leading order in the small parameters $\theta_{13}$ and $\cos 2 \theta_{23}$. The parameter $y$, which was introduced in [12], namely

$y=\frac{1}{4} \sin ^{2} 2 \theta_{12} \cos 2 \theta_{23}+\frac{1}{2} \sin 2 \theta_{12} \cos 2 \theta_{12} \theta_{13} \cos \delta$,

coincides at this order with $P_{1}$, while higher order terms are neglected. In [12], the errors on $P_{e \mu}$ and $P_{\mu \mu}$ were estimated to be 0.05 ; after 10 years, these errors amount to 0.03 and we see from Table 1 that, still, the uncertainty is mostly due to $P_{1}$. Equivalent parameters have been used also by other authors: e.g., in [17], we read that

A "universal" parameter related to B has been noted previously in the literature. It is called $-\Delta$ in $\mathrm{Z}$. $\mathrm{Z}$. Xing, Phys. Rev. D 74, 013009 (2006), and $+\Delta$ in W.

Rodejohann, JCAP 0701, 029 (2007).

although, curiously, Ref. [12] is not mentioned. Note that the parameter $y$ or equivalently $\Delta$ satisfies properties 2 and 4 of Sect. 2.1.

As already mentioned, a three-parameter description of the probabilities of vacuum oscillations with was first introduced in [17], where the parameters $A, B, C$ were defined: see Eq. (19) and note the symbol $\mathcal{O}\left(\epsilon^{3}\right)$ used there to emphasize the use of a quadratic expression in the small parameters that quantify the deviation from tribimaximal mixing, collectively denoted as $\epsilon$. This approximation is even better than the linear approximation, and thus was argued to be adequate for the present needs [17]. However, there are 

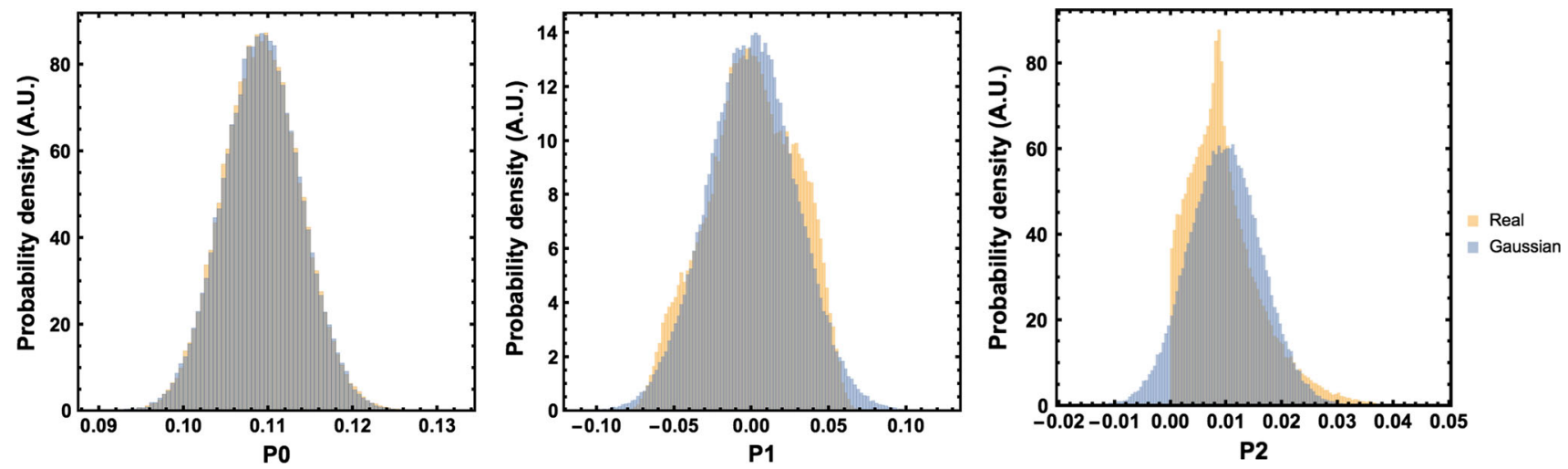

Fig. 1 Distribution of the natural parameters $P_{0}, P_{1}$ and $P_{2}$, due to the uncertainties in the mixing angles and the phase of leptonic CP violation

two evident shortcomings in the type of procedure adopted there to introduce the new parameterization: the emphasis on tribimaximal mixing given in Eq. (7), see also Eq. (16) of reference [17], is felt as artificial to date, especially now that the measurements showed that $\theta_{13}$ is non-zero, contradicting the most interesting prediction of tribimaximal mixing. Moreover, for the efforts made to obtain a quadratic the expansion is also unnecessary: the parameterization can be promoted without significant efforts to an exact one, as the expressions in Sect. 2.2 are valid to all orders in $\epsilon$ and easy to use. Thus, a direct, valid, and advantageous procedure is to introduce the parameterization since the start, as in Sect. 2.1. The detailed relation with our parameterization is

$A=9 P_{0}-1, \quad B=18 P_{1} \quad$ and $C=18 P_{2}$.

Both of them share features 1, 2, 3 discussed in Sect. 2.1 and they can be termed natural. In the following we adopt $P_{0}, P_{1}, P_{2}$ due to features 4 and 5 of Sect. 2.1 and to the fact that symbols reflect the hierarchy noted in Sect. 2.3. The parameterizations are, however, equivalent and it is easy to compare the results obtained with them.

Another equivalent parameterization was used in [18]. This begins from the "universal" parameter of [12] and improves the description of the oscillation probabilities by introducing a new parameter. Also this parameterization is introduced in connection to tribimaximal mixing and using the same quadratic expansion of the previous parameterization: note the symbol $\simeq$ of approximate equality in Eq. (2.5) of [18]. Thus, the same comments on the methodology made just above apply also to this case. The relation with our parameterization is

$\Delta=P_{1}$ and $\frac{\bar{\Delta}^{2}}{2}=P_{2}$.

A third parameter is not introduced, being replaced by $\theta_{12}$ and $\left|U_{e 3}\right|=\sin \theta_{13}$. Properties 2, 3, and 4 of Sect. 2.1 are all satisfied. Therefore, assumptions and results can be compared easily: e.g., Table 1 implies $P_{2}<0.017$ (resp., 0.031) at 1 sigma (resp., 3 sigma), whereas the value given in Eq. 16 of [18] implies $P_{2}<0.0145$ (resp., 0.0465). Similarly, the values $\Delta \simeq 0.02$ and $\bar{\Delta}^{2} \simeq 0.008$ quoted there correspond to $P_{1} \simeq 0.02$ and $P_{2} \simeq 0.004$, which are included in the 1 sigma range of Table 1 . The differences are due to the improved measurements of the oscillation parameters since 2007 and in particular to the inclusion of $\theta_{13}$, which now is measured and known to be non-zero. ${ }^{2}$ Note finally that in Fig. 1 of [17] the expected ranges of the parameters are presented, and these can be compared with our Fig. 1 and Table 1.

In the literature also other different linear combinations of the parameters have been considered; see e.g. [24]. The parameters $X, Y, Z$ introduced there, however, do not satisfy features 1, 2, 3 and 5 of Sect. 2.1, and in this technical and restricted sense, we do not call them 'natural'. The choice of parameters in [24] has its own motivations but it is less useful to keep under control the impact of the uncertainties on oscillations that is one of the main goal of the rest of this work.

\section{Applications}

We will consider two quantities that are affected by oscillations; the flavor ratios and the fraction of events due to Glashow resonance.

We denote the fractions of $v_{\ell}$ at source and the one at Earth (i.e., after oscillations), respectively, as

$\xi_{\ell}^{0}=F_{\ell}^{0} / \sum_{\ell} F_{\ell}^{0}$ and $\xi_{\ell}=F_{\ell} / \sum_{\ell} F_{\ell}$

where of course $\sum_{\ell} F_{\ell}^{0}=\sum_{\ell} F_{\ell}$. Suppose that the initial flavor ratio is given by

\footnotetext{
${ }^{2}$ Let us repeat that this differs from what was expected from the tribimaximal mixing scheme, which has been emphasized in $[17,18]$.
} 
$\left(\xi_{e}^{0}, \xi_{\mu}^{0}, \xi_{\tau}^{0}\right)=(1-g-h, g, h)$.

After propagation the flavor ratio is modified as follows:

$$
\begin{aligned}
\xi_{e}= & \frac{1}{3}+(2-3 g-3 h) P_{0}+(g-h) P_{1}, \\
\xi_{\mu}= & \frac{1}{3}+\frac{1}{2}(-2+3 g+3 h) P_{0} \\
& +(1-2 g-h) P_{1}+(g-h) P_{2}, \\
\xi_{\tau}= & \frac{1}{3}+\frac{1}{2}(-2+3 g+3 h) P_{0} \\
& +(-1+g+2 h) P_{1}-(g-h) P_{2} .
\end{aligned}
$$

Below, we will emphasize $\xi_{\mu}$ since it is quite directly connected to an observable quantity, namely, the fraction of track-type events.

\subsection{Flavor ratio after the oscillations}

A first application of the natural parameterization is the study of the flavor ratio of neutrinos, considering different mechanisms of production. ${ }^{3}$ The impact of uncertainties on the mixing angles and $\mathrm{CP}$-violating phase on the triangle of the flavors was first discussed in [7]. Here we will update the analysis by using updated values of the uncertainties. We will verify that a Gaussian treatment of the natural parameters leads to results in good agreement with Monte Carlo simulation and, moreover, we will compare our theoretical analysis with 3 years IceCube HESE data + passing muons.

We consider:

1. pions decay ( $h=0, g=2 / 3$; blue);

2. neutrons decay ( $h=0, g=0$; green);

3. damped muons ( $h=0, g=1$; red);

4. charm mesons ( $h=0, g=1 / 2$; orange).

Using Eq. (12), we represent the allowed regions by propagating the errors on the predictions by a Monte Carlo simulation; this gives the four dotted regions of the flavor triangles in Figs. 2 and 3.

These regions can be compared with those obtained with a Gaussian treatment of the errors on $P_{0}, P_{1}$, and $P_{2}$. Following the implementation of Appendix A, we obtain the four ellipses of Figs. 2 and 3 that enclose the $99 \%$ CL regions. We see that the differences between these the Monte Carlo and the Gaussian treatments are not very important. The Gaussian approach seems to be appropriate for the present needs. Note that the latter is significantly easier to implement.

Let us repeat that the expected theoretical regions (the dotted areas obtained by Monte Carlo and the elliptic curves corresponding to Gaussian approximation) depend only by

${ }^{3}$ Intermediate possibilities have also been considered in $[26,30]$.

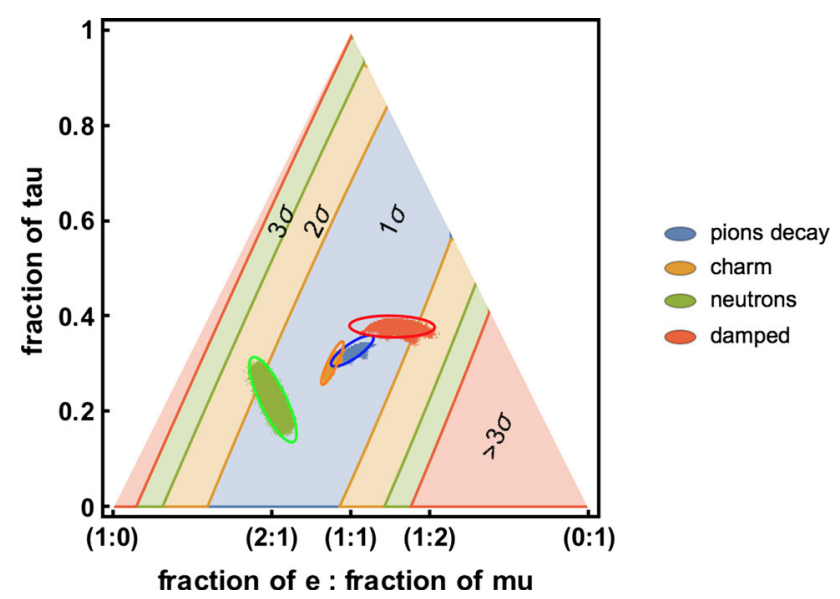

Fig. 2 Flavor triangle. The present observational information on the flavor composition of cosmic neutrinos at 1,2 , and $3 \sigma$ analyzed assuming $\alpha=2$ is compared with the expectations derived for four different hypotheses on the mechanism of production of the neutrinos. The ellipses derive from a Gaussian treatment of the error based on Table 1; see also Appendix A

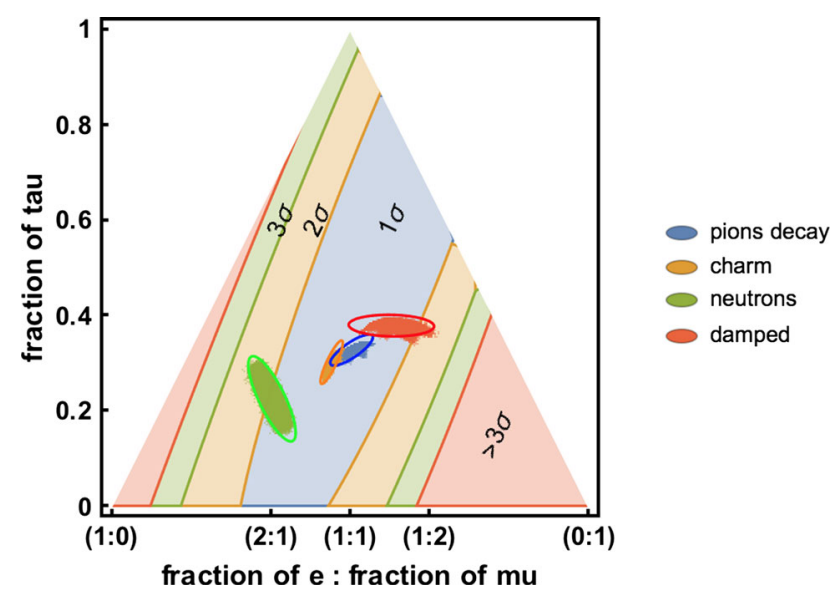

Fig. 3 Same as previous figure but using the spectral index $\alpha=2.3$ in the analysis of the IceCube data

initial flavor ratio and are not affected by the energy spectrum of the neutrinos, that is, assumed to be universal for all neutrinos. In other words, the four theoretical regions shown on the flavor triangles of Fig. 2 are just identical to those of Fig. 3.

On the contrary, the $1 \sigma, 2 \sigma$, and $3 \sigma$ zones do depend on the energy distribution of the neutrinos. The confidence levels, indicated in the flavor triangle, correspond to the result of the data analysis of IceCube events (the high-energy starting events, whose initial vertex is in the detectors and the passing muons, i.e. the through-going muons) discussed in [21,22]. They have been obtained assuming a power law distribution given by

$\frac{\mathrm{d} \phi}{\mathrm{d} E_{v}}=\phi_{0} E_{v}^{-\alpha}$. 


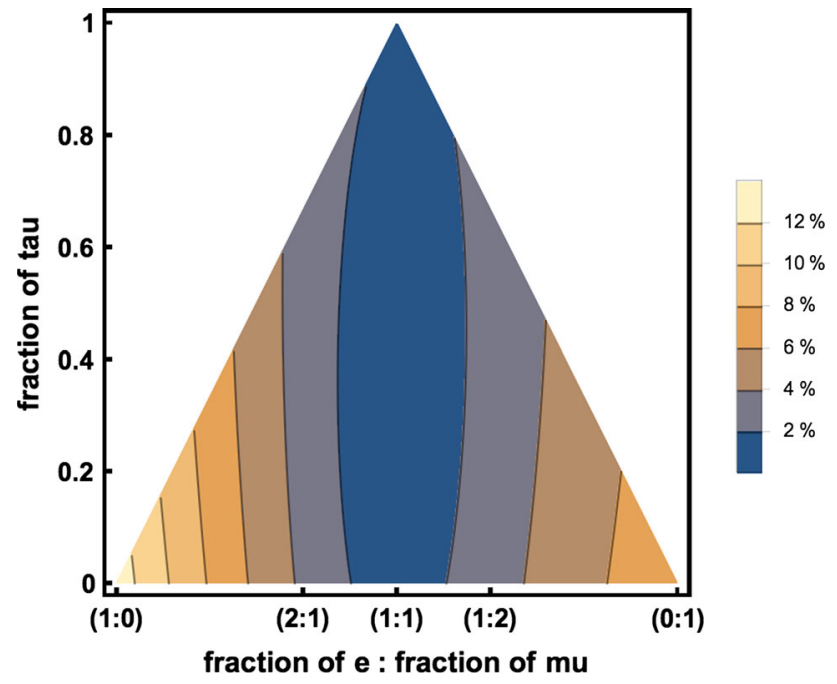

Fig. 4 Gaussian errors on the fraction of $v_{\mu}$ on Earth at 1 sigma, as a function of the neutrino fractions at the source

Thus, the observed flavor ratio is a function of the spectral index $\alpha$. In Fig. 2 we have used the value preferred by the simplest theoretical expectations, namely $\alpha=2$.

For comparison, we have shown also the case $\alpha=2.3$ in Fig. 3, namely the best fit value of the dataset of high-energy starting events collected by IceCube in the first three years. The comparison with Fig. 2 shows that the actual value of the spectral index plays some role in determining the allowed regions: the steeper spectrum $\alpha=2.3$ enhances the role of electron neutrinos and diminishes the one of muon neutrinos (whose effective area is very small close to the threshold); thus it requires one to increase the content of muon neutrinos at the source $\xi_{\mu}^{0}$ in order to reproduce the observed track-toshower ratio. For this reason, the agreement of the neutron decay scenario with the data worsens for $\alpha=2.3$. However, this kind of effects is not yet crucial for the analysis and in particular the neutron decay scenario is not yet excluded. In fact, the most important conclusion is just that the small number of events presently available, does not allow us yet to exclude any mechanism of production [21]. This remains true also using $\alpha=2.6$, namely the best fit including also low-energy events.

The confidence levels are in reasonable accordance with those of IceCube data analysis [22] (see again Fig. 2) and with those of [21]. The uncertainties due to the oscillation parameters have been presented in [21] in a different manner, but the results are in excellent agreement. ${ }^{4}$

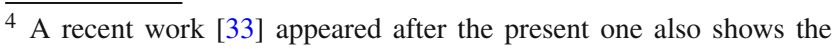
uncertainties due to oscillations in the flavor triangle. The Monte Carlo procedure is used and the results coincide with our ones.
}

\subsection{Errors on flavor ratio}

Let us discuss further the point of the errors. The flavor ratios given in Eq. 12 depend linearly by $P_{0}, P_{1}$, and $P_{2}$. Therefore, it is straightforward and quite easy to evaluate the Gaussian errors on these quantities. Let us focus on $\xi_{\mu}$.

From the formula for $\xi_{\mu}$, see again Eq. (12), we see that the term linear in $P_{1}$ becomes very small when $v_{e}$ and $v_{\mu}$ are about equal (e.g. charm mesons), and Fig. 4 confirms that that this type of mechanisms gives very small errors on the flavor ratio measured at Earth. Indeed, an initial composition of 1:1:1 would not be modified, or in other words, the error would be just null). On the contrary, the mechanisms that produce only $v_{e}$ (neutron decay) or only $v_{\mu}$ (damped muons) give the biggest error, about $10 \%$ on the final flavor ratio. The pion decay, which is the most plausible mechanism, is between the two extreme situations; the error on the muon fraction $\xi_{\mu}$ is about $3 \%$.

Let us remark that despite the relatively large uncertainties on $\theta_{23}$ and $\delta$ oscillations, the uncertainties on $\xi_{\mu}$ are small also in the worse scenario, namely, the neutron decay scenario.

These results are in good agreement with those of [18]; see in particular Fig. 4 there. Moreover, we note that the expectations from the pion decay mechanism agree quite well with the results of the analysis of the existing data; see again Fig. 2.

\subsection{Glashow resonance}

With the formalism of this paper it is easy to write analytical expressions for some interesting signal including the effect of three flavor oscillations. We analyze the case of Glashow resonance [25], i.e. the production of $W^{-}$starting from an electron antineutrino due to the process

$\bar{v}_{e}+e^{-} \rightarrow W^{-}$.

The process is possible when an antineutrino with energy greater than $6.3 \mathrm{PeV}$ collides with an electron at rest.

An interesting point for us is that different astrophysical mechanisms produce different fractions of $\bar{v}_{e}$, as already noted in [10] and further discussed in [18,26-28]. From the $p p$ interaction, e.g. [31], we can obtain all the types of pions; instead, from the $p \gamma$ interaction, e.g. [32], we obtain mostly $\pi^{+}$and $\pi^{0}$. After the decays the flavor ratio for $p p$ are approximatively equal for neutrinos and antineutrinos,

$\xi_{v}^{0}=(1,2,0) / 6, \quad \xi_{\bar{v}}^{0}=(1,2,0) / 6$,

while for $p \gamma$, the neutrino and antineutrino channels contain a different number of particles and lead to different flavors,

$\xi_{v}^{0}=(1,1,0) / 3, \quad \xi_{\bar{v}}^{0}=(0,1,0) / 3$, 


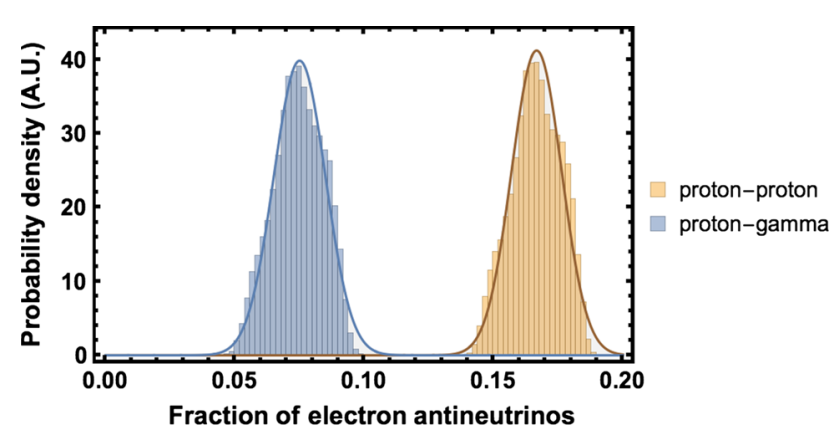

Fig. 5 Fraction of $\bar{v}_{e}$ due to the $p p$ or the $p \gamma$ interaction. The distribution obtained by a Monte Carlo extraction compares well with the Gaussian distributions obtained from Table 1 (continuous lines)

where we have normalized the two fluxes to a single particle. In the case of $p \gamma$ interaction $\bar{v}_{e}$ are not produced at the source, but only after the oscillations. The fraction of electron antineutrinos at Earth are given by a linear expression in the parameters $P_{0}$ and $P_{1}$ :

$\xi_{\bar{\nu}_{e}}^{p p}=\frac{1}{6}+\frac{1}{3} P_{1}$,

$\xi_{\bar{v}_{e}}^{p \gamma}=\frac{1-3 P_{0}}{9}+\frac{1}{3} P_{1}$.

These two distributions can be obtained with Monte Carlo simulation, using the distributions of mixing angle and $\mathrm{CP}$ phase violation. At $3 \sigma$ (i.e., $99.7 \%$ ) we find that

$\xi_{\bar{v}_{e}}^{p p}=0.167 \pm 0.029$,

$\xi_{\bar{\nu}_{e}}^{p \gamma}=0.075 \pm 0.029$,

where the uncertainty is due to $P_{1}$. These ranges compare well with the maximum and minimum values given in Fig. 6 of [18]. Note that we are using the newest measurements of the oscillation parameters and in particular we have included the effect of $\theta_{13}$, which is now measured and known to be non-zero.

If the total flux of neutrinos is the same in both cases, this implies that the signal from the Glashow resonance is about two times weaker in the $p \gamma$ case than in the $p p$ case. Thus, oscillations give a final flavor ratio that is significantly different in the case of $p \gamma$-production mechanism. The results are further illustrated in Fig. 5. Note that, also for this application, the very small differences from the result of the Gaussian approximation, evident from Fig. 5, justify the use of a linear approach.

If we compare the two cases assuming to have the same cosmic ray flux and the same amount of collisions, as done in [10], the difference between the two cases is even more dramatic. In fact, the $p \gamma$ mechanism leads to neutrinos of lower energies than those from the $p p$ mechanism, and thus the chances of observing a neutrino signal from Glashow resonance decreases.

\section{Summary and discussion}

The results of IceCube have greatly increased the interest in an accurate description of propagation of cosmic neutrinos, accounting in particular for the minimal hypothesis of three flavor oscillation in vacuum. In this work we have discussed what is the natural choice of the parameters to describe vacuum oscillations of cosmic neutrinos.

We have shown that the parameterization of neutrino oscillations in vacuum introduced in [17] can be promoted to an exact one (without any need of performing expansions, approximations or making references to the case of tribimaximal neutrino mixing) and can be argued to be the natural parameterization for the discussion of oscillations of cosmic neutrinos; see Sect. 2.1. We have obtained new and exact expressions for the three relevant parameters in terms of the standard mixing parameters; see Sect. 2.2.

In Sect. 3, we have illustrated the usefulness of the natural parameters $P_{0}, P_{1}, P_{2}$ given in Eq. 2 by discussing the expectations on the neutrino flavor ratios and their errors. We have also analyzed the expectations on the intensity of the signal due to Glashow resonance, which depends on the mechanism of neutrino production. We have included the effect of the uncertainties on oscillation in the flavor triangle, comparing the predictions with the results of the analysis of the flavor of cosmic neutrinos seen by IceCube, finding results in good agreement with the previous literature; see [21] and [22]. We have confirmed [18] that the $p p$ and $p \gamma$-production mechanisms lead to significantly different predictions for the intensity of Glashow resonance.

With improved data and analyses of IceCube data, it will be more and more important to include the effect of the various theoretical uncertainties, including those due to parameters of neutrino oscillations. Here, we have shown that the expectations obtained with a Gaussian treatment of the natural parameters $P_{0}, P_{1}, P_{2}$ are very similar to those obtained in more complete descriptions of three flavor oscillations based on [21]. We have proved that the Gaussian treatment is particularly easy to implement and is quite adequate for the present needs.

Let us conclude by stressing that the parameters $P_{0}, P_{1}$, and $P_{2}$ can be used also to provide us with an ideally exact and compact description of cosmic neutrino oscillations. E.g., the distributions of these parameters obtained with Monte Carlo methods, described above and illustrated in the flavor triangles of Figs. 2 and 3, go beyond the Gaussian approximation and they are formally accurate.

Future global analyses of the data on three flavor neutrino oscillation will be able to derive which are the precise distributions of the natural parameters of the cosmic neutrino oscillations and their correlations, simply because these parameters are functions of the conventional mixing angles and CP-violating phase. In view of the above discussion, it 
will be particularly useful to have precise distributions of the parameters $P_{1}$ and $P_{2}$.

Acknowledgments We are grateful to F. Aharonian, G. Pagliaroli and F.L. Villante for useful discussions and collaboration on related matter. We thank an anonymous referee for pointing out to us the first reference on Glashow resonance and cosmic neutrino oscillations; F.V. gladly acknowledges a discussion of the same topic with Klas Hultqvist at the Neutrino Telescope conference at Venice (March 2015).

Open Access This article is distributed under the terms of the Creative Commons Attribution 4.0 International License (http://creativecomm ons.org/licenses/by/4.0/), which permits unrestricted use, distribution, and reproduction in any medium, provided you give appropriate credit to the original author(s) and the source, provide a link to the Creative Commons license, and indicate if changes were made. Funded by SCOAP ${ }^{3}$.

\section{Appendix A: Allowed regions in the Gaussian approximation}

Let us consider the two dimensional Gaussian likelihood,

$$
\begin{aligned}
& \mathcal{L}=\frac{\exp \left[-\frac{1}{2}(\vec{v}-\langle\vec{v}\rangle)^{t} \Sigma^{-2}(\vec{v}-\langle\vec{v}\rangle)\right]}{2 \pi \sqrt{\operatorname{det}\left(\Sigma^{2}\right)}} \quad \text { where } \vec{v}=\left(\begin{array}{l}
x \\
y
\end{array}\right), \\
& \Sigma^{2}=\left(\begin{array}{cc}
\sigma_{x}^{2} & \sigma^{2} \\
\sigma^{2} & \sigma_{y}^{2}
\end{array}\right) \text {. }
\end{aligned}
$$

A confidence level $(0<C . L .<1)$ defines the allowed region $\mathcal{L}>\mathcal{L}_{\max }(1-C$.L. $)$. Its contour is an ellipse that can be obtained from the following parametric expression:

$$
\begin{aligned}
\left(\begin{array}{l}
x(\varphi) \\
y(\varphi)
\end{array}\right)= & \left(\begin{array}{l}
\langle x\rangle \\
\langle y\rangle
\end{array}\right)+\sqrt{-2 \log (1-C \cdot L .)}\left(\begin{array}{cc}
\cos \theta & \sin \theta \\
-\sin \theta & \cos \theta
\end{array}\right) \\
& \times\left(\begin{array}{c}
\sigma_{+} \\
\sigma_{-} \sin \varphi
\end{array}\right)
\end{aligned}
$$

where $\varphi=[0,2 \pi]$ and where we defined

$$
\begin{aligned}
& \theta=\frac{1}{2} \arctan \left[\frac{2 \sigma^{2}}{\sigma_{y}^{2}-\sigma_{x}^{2}}\right], \\
& \sigma_{ \pm}^{2}=\frac{2\left(\sigma_{x}^{2} \sigma_{y}^{2}-\sigma^{4}\right)}{\sigma_{x}^{2}+\sigma_{y}^{2} \pm\left(\sigma_{y}^{2}-\sigma_{x}^{2}\right) \sqrt{1+\tan ^{2} 2 \theta}} .
\end{aligned}
$$

In the flavor triangle, we have known linear combinations of $P_{0}, P_{1}, P_{2}$,

$$
x=\left(\xi_{\mu}-\xi_{e}\right) / \sqrt{3} \equiv x_{0}+x_{i} P_{i} \quad \text { and } y=\xi_{\tau} \equiv y_{0}+y_{i} P_{i} .
$$

From Table 1, one evaluates $\langle x\rangle=x_{0}+x_{i}\left\langle P_{i}\right\rangle, \sigma_{x}^{2}=x_{i}^{2} \delta P_{i}^{2}$, $\sigma^{2}=x_{i} y_{i} \delta P_{i}^{2}$, etc.

\section{References}

1. Aartsen MG, et al. [IceCube Collaboration]. First observation of PeV-energy neutrinos with IceCube. Phys. Rev. Lett. 111:021103 (2013)

2. Aartsen MG, et al. [IceCube Collaboration]. Evidence for highenergy extraterrestrial neutrinos at the IceCube Detector. Science 342:1242856 (2013)

3. Aartsen MG, et al. [IceCube Collaboration]. Observation of highenergy astrophysical neutrinos in three years of IceCube data. Phys. Rev. Lett. 113:101101 (2014)

4. S.M. Bilenky, B. Pontecorvo, Lepton mixing and neutrino oscillations. Phys. Rep. 41, 225 (1978)

5. J.G. Learned, S. Pakvasa, Detecting tau-neutrino oscillations at PeV energies. Astropart. Phys. 3, 267 (1995)

6. H. Athar, M. Jezabek, O. Yasuda, Effects of neutrino mixing on high-energy cosmic neutrino flux. Phys. Rev. D 62, 103007 (2000)

7. G. Barenboim, C. Quigg, Neutrino observatories can characterize cosmic sources and neutrino properties. Phys. Rev. D 67, 073024 (2003)

8. Beacom JF, et al. Measuring flavor ratios of high-energy astrophysical neutrinos. Phys. Rev. D 68:093005 (2003) [E-ibid. D 72 (2005) 019901]

9. P.D. Serpico, M. Kachelriess, Measuring the 13-mixing angle and the CP phase with neutrino telescopes. Phys. Rev. Lett. 94, 211102 (2005)

10. L.A. Anchordoqui, H. Goldberg, F. Halzen, T.J. Weiler, Neutrinos as a diagnostic of high energy astrophysical processes. Phys. Lett. B 621, 18 (2005)

11. V.S. Berezinsky, A.Z. Gazizov, Cosmic neutrinos and possibility to search for $\mathrm{W}$ Bosons having $30-\mathrm{GeV}-100-\mathrm{GeV}$ masses in underwater experiments. JETP Lett. 25, 254 (1977)

12. M.L. Costantini, F. Vissani, Expected neutrino signal from supernova remnant RX J1713.7-3946 and flavor oscillations. Astropart. Phys. 23, 477 (2005)

13. Vissani F. Neutrinos from supernova remnants after the first H.E.S.S. observations. Published in the proceedings of Vulcano workshop 2006 frontier objects in astrophysics and particle physics. Giovannelli F, Mannocchi G, editors. arXiv:astro-ph/0609575

14. F.L. Villante, F. Vissani, How precisely neutrino emission from supernova remnants can be constrained by gamma ray observations? Phys. Rev. D 78, 103007 (2008)

15. F. Vissani, F. Aharonian, Galactic sources of high-energy neutrinos: highlights. Nucl. Instrum. Methods A 692, 5 (2012)

16. F. Vissani, G. Pagliaroli, F.L. Villante, The fraction of muon tracks in cosmic neutrinos. JCAP 1309, 017 (2013)

17. S. Pakvasa, W. Rodejohann, T.J. Weiler, Unitary parametrization of perturbations to tribimaximal neutrino mixing. Phys. Rev. Lett. 100, 111801 (2008)

18. S. Pakvasa, W. Rodejohann, T.J. Weiler, Flavor ratios of astrophysical neutrinos: implications for precision measurements. JHEP 0802, 005 (2008)

19. L. Fu, C.M. Ho, T.J. Weiler, Aspects of the flavor triangle for cosmic neutrino propagation. Phys. Rev. D 91, 053001 (2015)

20. Palomares-Ruiz S, Vincent AC, Mena O. Spectral analysis of the high-energy IceCube neutrinos. arXiv:1502.02649 [astro-ph.HE]

21. A. Palladino, G. Pagliaroli, F.L. Villante, F. Vissani, What is the flavor of cosmic neutrinos seen by IceCube? Phys. Rev. Lett. 114(17), $171101(2015)$

22. Aartsen MG, et al. [IceCube Collaboration]. Flavor ratio of astrophysical neutrinos above $35 \mathrm{TeV}$ in IceCube. Phys. Rev. Lett. 114(17): 171102 (2015)

23. O. Mena, S. Palomares-Ruiz, A.C. Vincent, Flavor composition of the high-energy neutrino events in IceCube. Phys. Rev. Lett. 113(9), 091103 (2014) 
24. X.J. Xu, H.J. He, W. Rodejohann, Constraining astrophysical neutrino flavor composition from leptonic unitarity. JCAP 1412(12), 039 (2014)

25. S.L. Glashow, Resonant scattering of antineutrinos. Phys. Rev. 118, $316(1960)$

26. A. Bhattacharya, R. Gandhi, W. Rodejohann, A. Watanabe, The Glashow resonance at IceCube: signatures, event rates and $p p$ interactions. JCAP 1110, 017 (2011)

27. V. Barger, L. Fu, J.G. Learned, D. Marfatia, S. Pakvasa, T.J. Weiler, Glashow resonance as a window into cosmic neutrino sources. Phys. Rev. D 90(12), 121301 (2014)

28. Z.Z. Xing, S. Zhou, The Glashow resonance as a discriminator of UHE cosmic neutrinos originating from p-gamma and p-p collisions. Phys. Rev. D 84, 033006 (2011)

29. M.C. Gonzalez-Garcia, M. Maltoni, T. Schwetz, Updated fit to three neutrino mixing: status of leptonic CP violation. JHEP 1411, 052(2014)
30. A. Chatterjee, M.M. Devi, M. Ghosh, R. Moharana, S.K. Raut, Probing CP violation with the first three years of ultrahigh energy neutrinos from IceCube. Phys. Rev. D 90(7), 073003 (2014)

31. Kelner SR, Aharonian FA, Bugayov VV. Energy spectra of gammarays, electrons and neutrinos produced at proton-proton interactions in the very high energy regime. Phys. Rev. D 74:034018 (2006) [Erratum-ibid. D 79 (2009) 039901]

32. Kelner SR, Aharonian FA. Energy spectra of gamma-rays, electrons and neutrinos produced at interactions of relativistic protons with low energy radiation. Phys. Rev. D 78:034013 (2008) [Erratum-ibid. D 82 (2010) 099901]

33. Argelles CA, Katori T, Salvado J. arXiv:1506.02043 [hep-ph] 